\title{
Eisenmenger syndrome with transposition of pulmonary veins in Pomeranian: a case report
}

\author{
Georgi I. Georgiev
}

Department of Anatomy, Histology and Physiology, Faculty of Veterinary Medicine, University of Forestry, Sofia, Bulgaria;

\begin{abstract}
Studies of congenital cardiac abnormalities and vascular malformations, as well as their breed predispositions in domestic animals, have significantly increased recently. The purpose of this case report is to present to vets the Eisenmenger syndrome in combination with a vascular malformation in a Pomeranian, which is extremely interesting for the teratology of the dog. Radiography was performed and therapy was assigned, but a quick fatal outcome followed. Then the patient underwent a pathological anatomy examination with precise macro-dissection. We hope that thorough this message we will draw attention to different severe congenital heart abnormalities in toy breeds and their early diagnosis.
\end{abstract}

Keywords: dog, Eisenmenger syndrome, heart defects, transposition of pulmonary veins.

\section{Background}

Heart malformations are more common in dogs and cattle than in horses and cats (1). Clinically they are classified as cyanotic and acyanotic depending on whether they are associated with cyanosis in postnatal life, which is presented with purplish to bluish tissue with a dense surface capillary network, seen most easily in examination of the oral mucosa, gums (1), or vulval and prepucial mucous membranes (1). Eisenmenger syndrome is a severe cardiac anomaly similar to the Tetralogy of Fallot and is characterized by elevated pulmonary vascular resistance and left-to-right shunting of blood from the systemic to the pulmonary circulation associated with patent ductus arteriosus, ventricular septal $(1,2)$, atrial septal, and 
aortic-pulmonary septal defect (1). This additional source of blood causes dilation and hypertrophy of the right ventricle. The significant left-right shunt increases the blood flow in the pulmonary blood vessels where increased pressure often leads to pulmonary vascular obstructive disease, leading to increase in pulmonary vascular resistance $(1,2,3,4,5,6)$. The left to right shunting cardiac defect are described as changes from ventral septal defect and patent ductus arteriosus, causes pulmonary overperfusion, leading to pulmonary hypertension and eventually reversed shunting (right to left) as the right heart pressures increase and become equal to or greater than systemic arterial pressures (4). Due to this blood path the systemic resistance is exceeded, the shunt is reversed and cyanosis appears. Right ventricular hypertrophy appears because it must overcome the high pulmonary vascular resistance $(1,2,3,4,5,6)$. Transposition of great vessels or reversal of systemic, pulmonary outflow and inflow are rarely found in animals (1). Normally pulmonary and systematic circulation are separated and closed off, which is required for postnatal survival. For the fetus to survive such anomalies must be combined with atrial and ventricular septal defects associated with passable ductus arteriosus, but even with such anatomical compensations the quality of blood in the body remains quite unacceptable (1).

\section{Case description}

A 4-month-old female dog, Pomeranian breed (Toy spitz). The owners observed a weak growth, weakness and easy fatigue, seizures (syncope) and shortened breathing. The clinical examination established dyspnea, cyanosis developments on a mild exertion in the oral mucosa and gums, as well as an unusual cardiac murmur. In the blood test erythrocytosis was observed, while radiography in lateral projection revealed cardiomegaly that was more prominent in the right part of the heart and apparent main pulmonary arteries segment (Fig.1). Despite the therapy assigned and a temporary, insignificant improvement of the condition of the dog, a lethal outcome was observed. Due to the interesting symptoms after the lethal outcome this animal was admitted at the University Clinic of the Faculty of Veterinary Medicine, University of Forestry, Sofia, for an expert autopsy. A precise pathological anatomy dissection was performed, which identified several cardiac malformations.

During the autopsy of the abdominal organs changes were not observed. The enlarged heart from the radiographic finding was confirmed after opening of the thoracic cavity (Fig. 2a). Transposition of pulmonary veins, evacuating the blood from the right lung (Fig. 2a), as well as their abnormal ending in the right atrium was observed (Fig. 2b). On the left a passable or persistent ductus arteriosus (ductus Botali) was observed (Fig. 3), linking the pulmonary trunk to the aortic arch, representing a congenital vascular malformation. After removal and resection of the heart a persistent foramen ovale or atrial septal defect, linking both atria, was observed (Fig. 4), as well as a pronounced defect of the interventricular septum, shaped like a channel (Fig. 5). Together with these malformations hypertrophy of the right ventricle wall was confirmed (Fig. 6a), characteristic of the Eisenmenger syndrome and the Tetralogy of Fallot.

Additionally, hypertrophy of the left ventricle wall was observed (Fig. 6b), where such finding is not specific for this syndrome. 


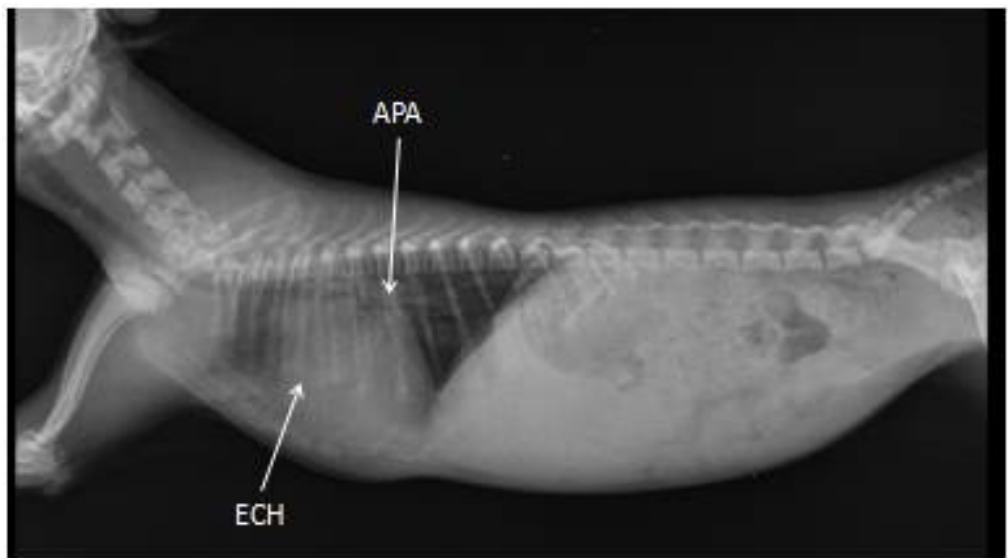

Fig. 1. Fig. 1. Radiographic view in lateral projection of the dog $-\mathrm{ECH}-$ enlarged right atrium and ventricle; APA - apparent main pulmonary arteries

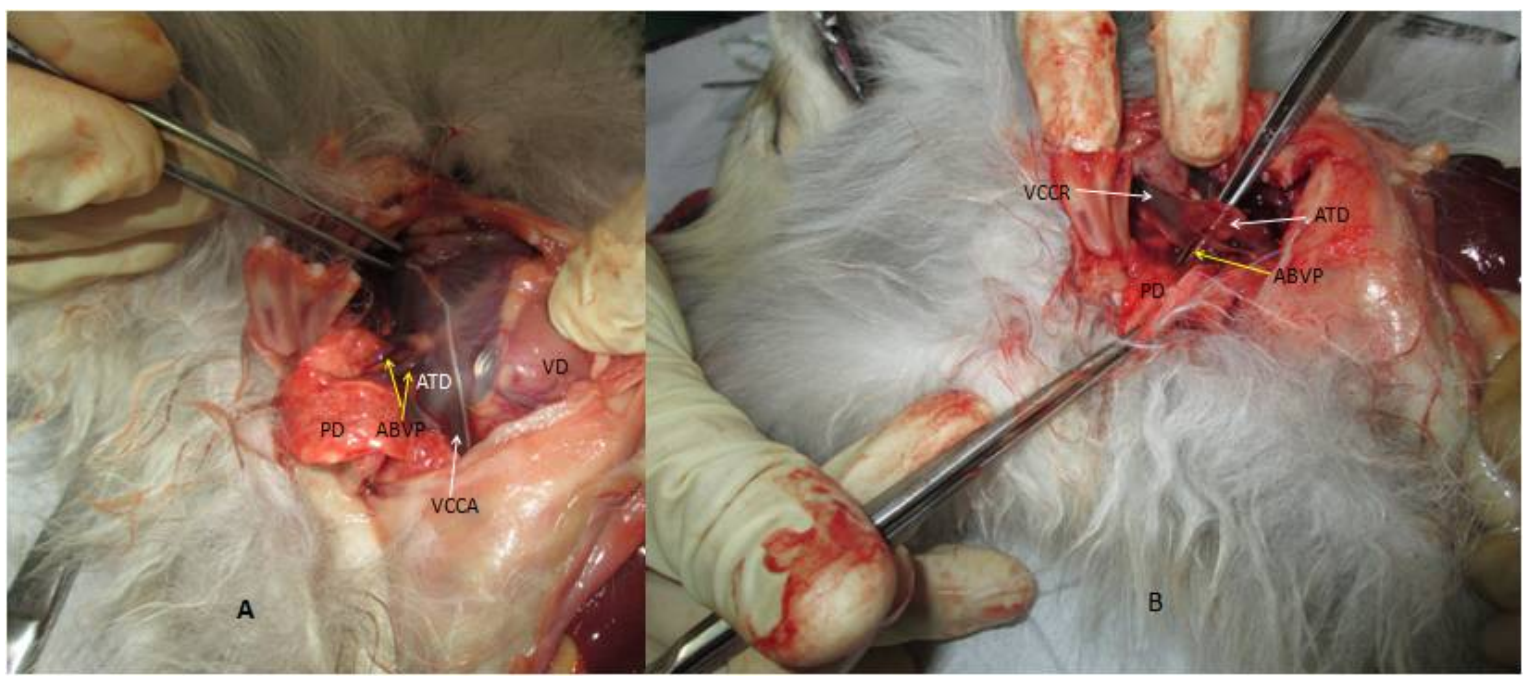

Fig. 2. Right side of the thoracic cavity of the dog, not opened (A) and opened (B) right atrium ATD - right atrium; VD- right ventricle; VCACR - cranial vena cava; VCCA-caudal vena cava; PD - right lung; ABVP - abnormal pulmonal veins. 


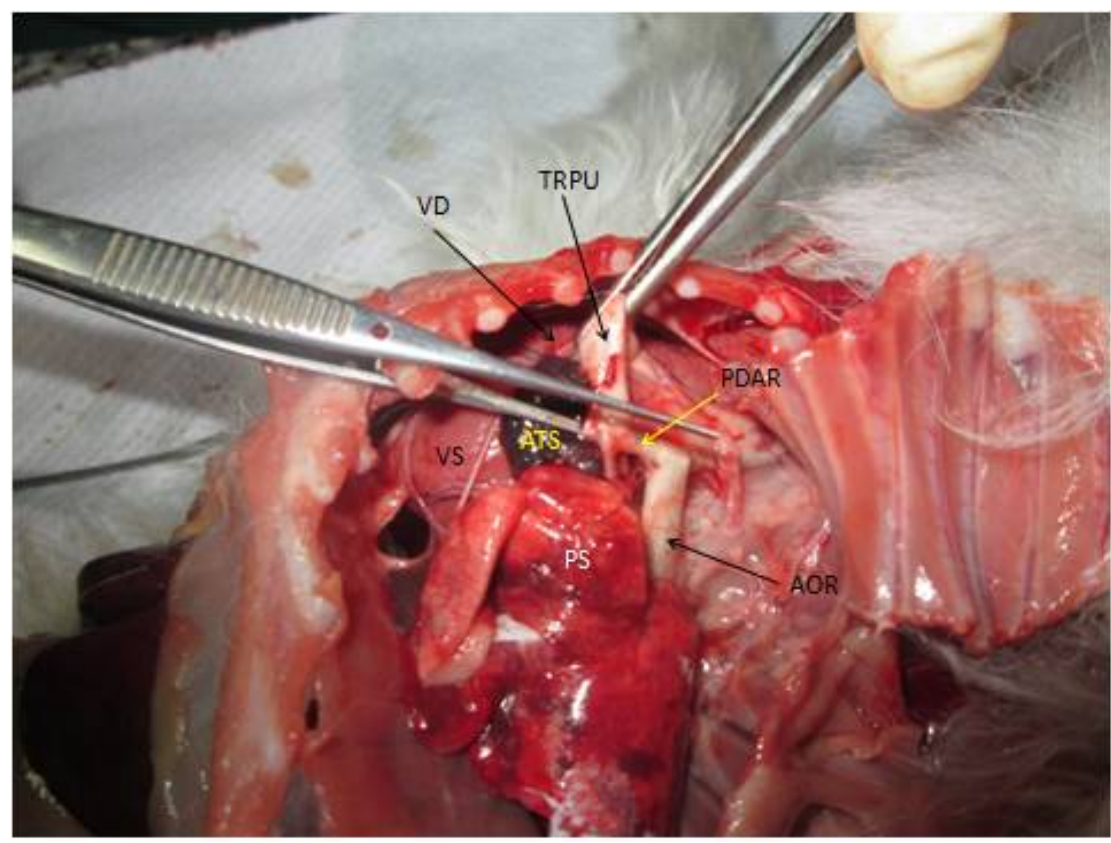

Fig. 3. Left side of the thoracic cavity of the dog - VS - left ventricle; ATS - left atrium; VD - right ventricle; TRPU - pulmonary trunk; AOR - aortic arch; PDAR - patent ductus arteriosus; PS - left lung.

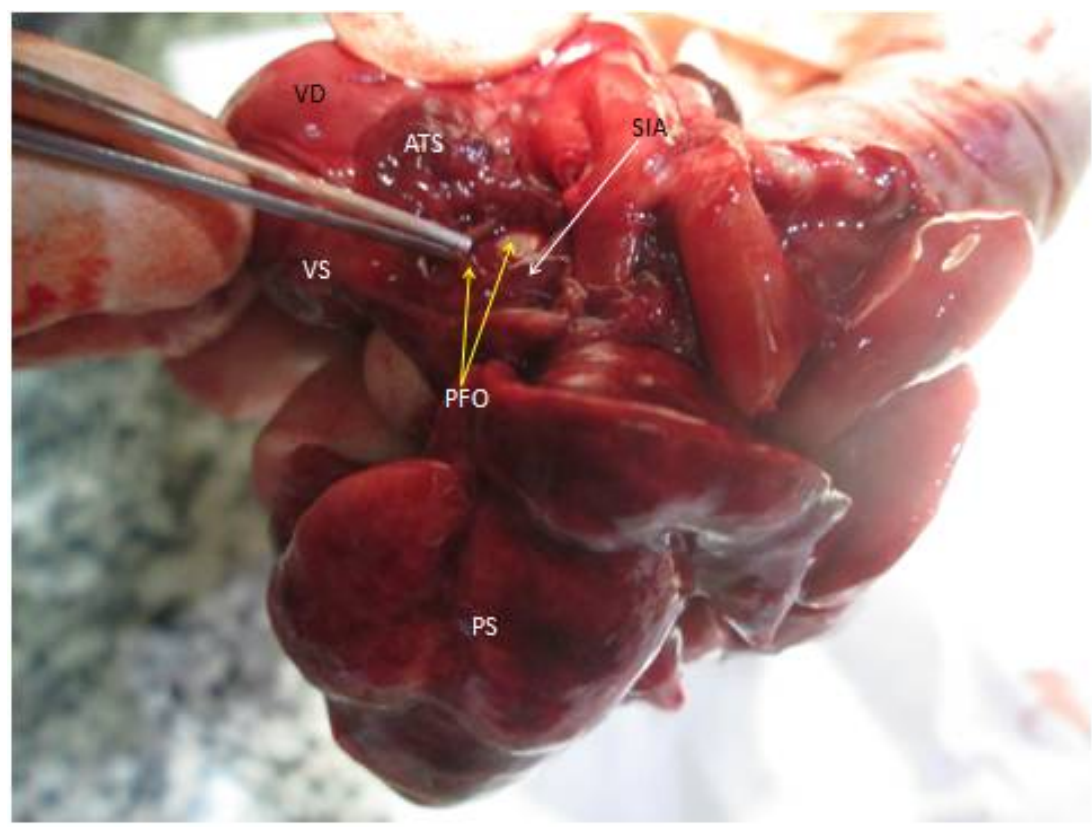

Fig. 4. Heart and lungs of the dog (removed) - VS - left ventricle; ATS - left atrium; VD- right ventricle; PS - left lung; SIA - septum interatriale; PFO- persistant foramen ovale. 


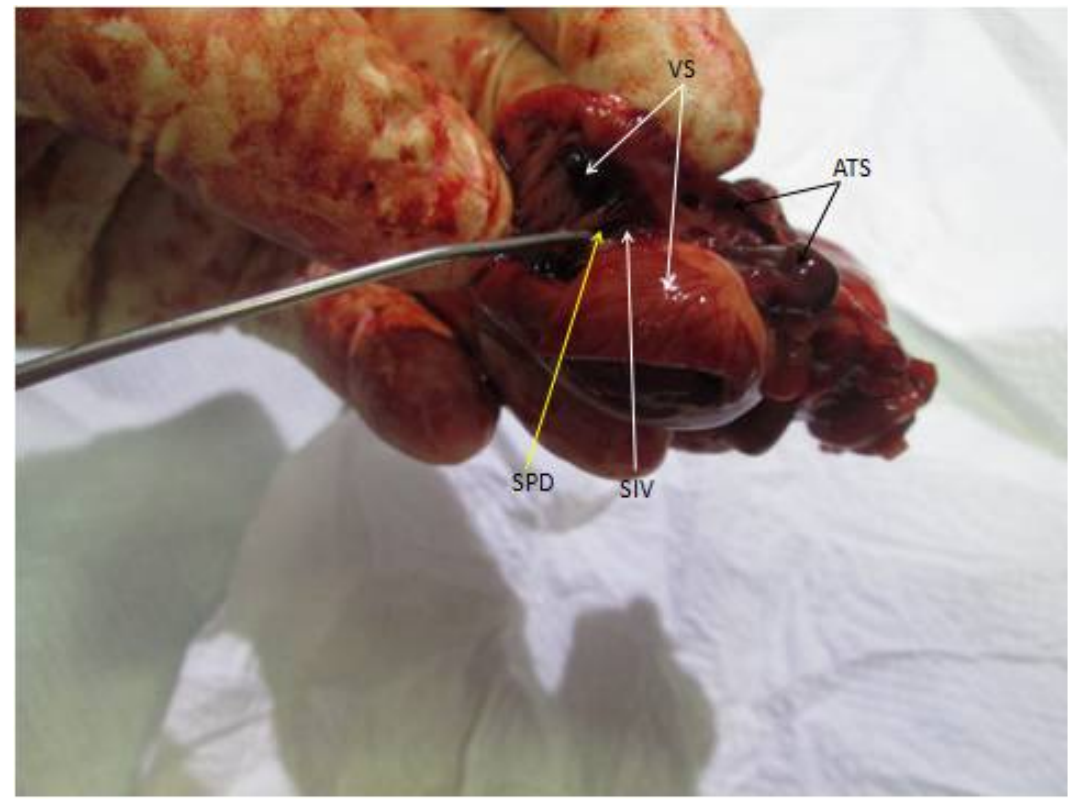

Fig. 5. Heart and lungs of the dog (removed) - VS - left ventricle; ATS - left atrium; SIV - septum interventriculare; SPD - interventricular septal defect.

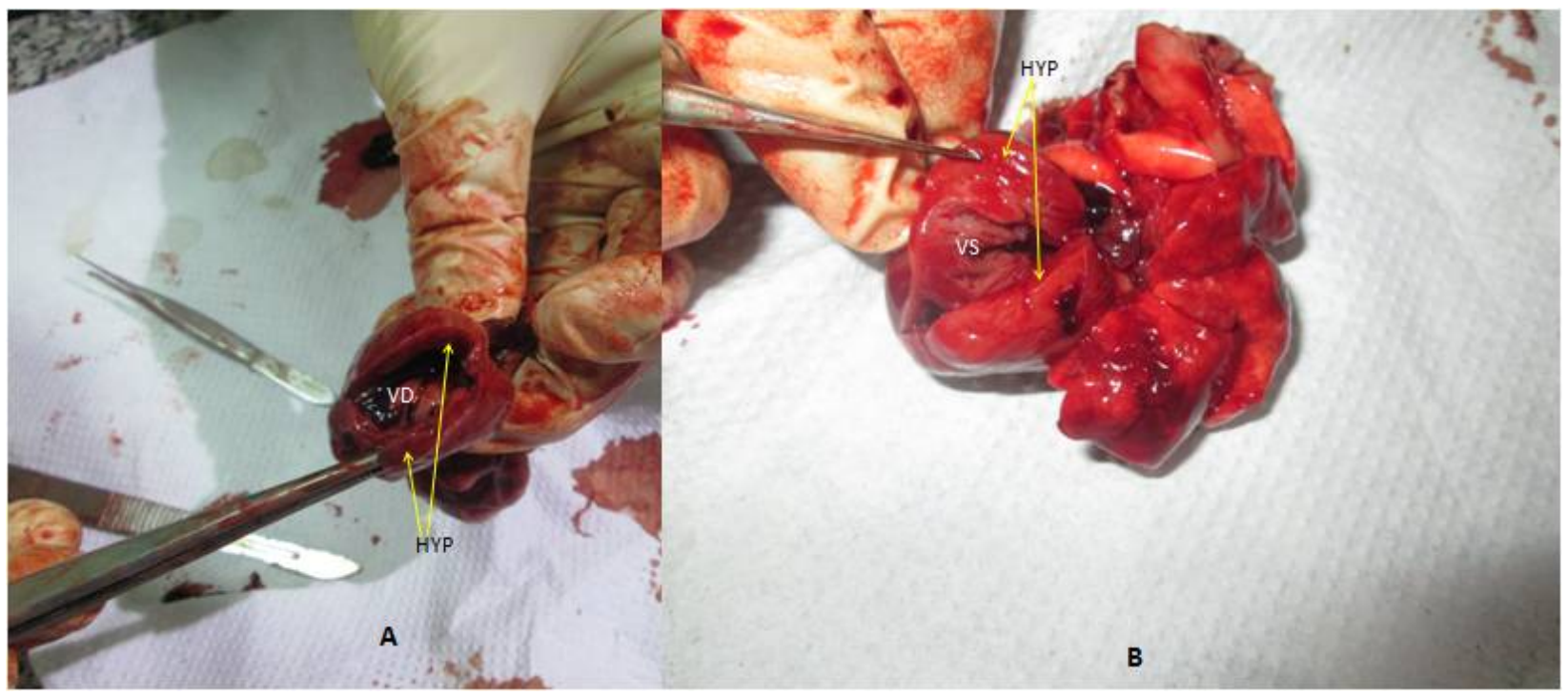

Fig. 6. Right (A) and left (B) side of the heart - VS - left ventricle; VD - right ventricle; HYP - right ventricle hypertrophy; HYP - left ventricle hypertrophy.

\section{Discussion}

With such severe cardiac abnormalities with prominent clinical signs animals live in most cases less than a year. Cases of survivors up to 3 years have been described, but dogs with such conditions have 
characteristically higher mortality at a young age $(3,5)$. With the severe heart malformations, presenting with cyanosis and various defects, the finding observed resembles the most the Eisenmenger syndrome in dogs, where patent ductus arteriosus, atrial septal and interventricular defect, hypertrophy of the right ventricle $(1,2)$ and aortic-pulmonary defect $(1)$, (which was the only one not observed in this dog) are characteristic. During necropsy, the proximal main pulmonary artery is dilated in dogs. The small pulmonary vasculature is abnormal. Dilation of the large elastic pulmonary arteries is present (2). The smaller elastic arteries are with hypertrophied walls and large lumina. Into muscular arteries are marked increase in connective tissue and fibroblasts, focal subintimal proliferations which often obliterats the vessel lumen, just like medial muscular hypertrophy with severe disruption of the internal and external elastic laminae, and plexiform lesions (2). Eisenmenger's syndrome has been reported in a 9-month-old Border Collie (7) and in a 2-year-old Shiba Inu dog (8). This syndrome is similar to the Tetralogy of Fallot in dogs, where interventricular defect and hypertrophy of the right ventricle are also observed, presenting as cyanotic heart malformation, but differs with overriding aorta and pulmonary opening stenosis (1, 3, 5, 9). Some breeds are predisposed to the Tetralogy of Fallot, like the English Bulldog, Keeshond, Miniature Poodle, Miniature Schnauzer and Wirehaired Fox Terrier, as well as cats, calves and foals $(1,5,9,10)$.

When these four malformations are combined with atrial septal defect this is another more severe abnormality, called Pentalogy of Fallot, which is described in calves (11).

From a differential diagnostic point of view this case must be distinguished from acyanotic cardiac malformations, such as atrial and ventricular septal defect. They can be independently developed, as well as in combination with other abnormalities. The large ventricular septal defect is part of the Eisenmenger syndrome, where a reverse shunt is present and pulmonary vascular disease and pulmonary hypertension develop (1). This anomaly as confirmed by other authors, manifests in the early stages of life and rarely in small dog breeds $(3,5)$. According to the localization other authors classify ventricular defects in four types - membranous, outlet infundibular, inlet channel and muscular-trabecular (1). In this Pomeranian case the malformation is determined as inlet channel. Independent interventricular anomalies are observed in the breeds English Bulldog, Keeshond, English Springer Spaniel, Beagle, Chow Chow, Newfoundland, Samoyed and all domestic animals with acyanotic presentation $(1,5,9,10)$. If the defect is small (restrictive ventricular septal defect), it is manifested with little or no functional disturbances due to the minimally increased pulmonary blood flow, as described in many cases in cattle (1). In cases of aggravated defect, as seen with this Pomeranian, right ventricular dilation is presented with subsequent hypertrophy. Very often ventricular septal defects are accompanied by other heart malformations, and then cyanotic signs occur secondarily (1).

Atrial septal defects alone are described in Boxer, Samoyed, Old English Sheepdog, Doberman Pinscher, and cats $(1,5,9,10)$. They are also present in $20 \%$ of pigs and calves but without any functional changes (12). This defect occurs when the septum secundum overlays one or several openings of septum primum, which leads to overwork and overload of the right side of the heart, and then leads to dilatation and hypertrophy (1). In failure to close foramen ovale, however, hypertrophy of the left ventricle is possible (1), which also explains our finding in this case. This atrial defect is very often combined with other cardiac malformations.

In an independent patent ductus arteriosus the symptoms are extremely variable (1), but again overloaded pulmonary blood flow and circulation are observed, where again dilatation and hypertrophy of the left atrium and ventricle are observed, which further confirms and explains the described by us. Such 
pathology has been reported in the Miniature Poodle, German Shepherd, Collie, Shetland Sheepdog, Pomeranian, Cocker Spaniel, English Springer Spaniel, Maltese, Yorkshire Terriers and other toy breeds and cats $(1,5,9,10,13)$.

Transposition of the systemic and pulmonary outlet and inlet vessels is significantly rare in animals. Its presence usually leads to very quick death shortly after birth. This malformation is usually combined with atrial and ventricular septal defect associated with passable ductus arteriosus (1). Compensatory mechanisms of all these heart abnormalities explain the survival of the observed dog until the fourth month, as well as the initial improvement from the therapy assigned in our clinical case.

From everything described above it is most important to mention that the differential diagnosis of the described case is close in terms of clinical presentation and radiological findings to the tetralogy of Fallot, but in order to differentiate it we would recommend using imaging diagnostic methods such as echocardiography, Doppler ultrasound, and angiocardiography for identification and specification of existing cardiac malformations (1, 4, 5, 9). Split second heart sound (S2) caused by pulmonary hypertension and delayed pulmonary valve closure helps differentiate the described condition from Tetralogy of Fallot where split S2 is generally absent (4).

Treatment with sildenafil citrate may help alleviate secondary pulmonary hypertension of the dog (14) and cat (15). Surgical closure of a right-to-left shunting ventricular septal defect is generally contraindicated. Phlebotomy to relieve severe polycythemia administration may be tried to improve clinical signs (2).

\section{Conclusion}

Finally, through this case of a rare combination of heart and vascular anomalies in a dog from the Pomeranian breed we hope to draw the attention of practitioner veterinarians to more careful research in heart malformations in order to determine the distribution, breed predisposition, diagnostics and eventual surgical treatment of the Eisenmenger syndrome in the dog.

\section{Acknowledgements}

The author would like to express his gratitude to Assoc. prof. Vasil Manov, DVM, PhD, Department of "Internal non transmissive disease, pathology and pharmacology" Faculty of Veterinary medicine, University of Forestry for providing this case.

\section{References}

1. Sinowatz, F. Teratology. In: Essential of Domestic Animal Embryology. (Hyttel, P., F. Sinowatz, M. Vejlsted, K. Betteridge, Eds). Saunders and Elsevier; Edinburgh, London, New York, Oxford, Philadelphia, St Louis, Sydney, Toronto, 2010; pp. 347-352.

2. Kittleson, M.D., R. D. Kienle. Small Animal Cardiovascular Medicine. Mosby Inc., 1998. 
3. Bonagura, J.D. Congenital heart disease. In: Textbook of veterinary internal medicine. (Ettinger, S.J., Eds), 3rd ed. W.B. Saunders Company, Philadelphia, London, Toronto, 1989.

4. Brownlie, S. Eisenmenger`s syndrome. Vetlexicon Canis, Vetstream Ltd., 2014.

5. Tilley, L.P., F.W.K. Smith Jr. The 5 minute veterinary consult canine and feline. Williams and Wilkins Company, Baltimore, 1997.

6. Tilley, L.P., J.M. Owens. Congenital heart defects. In: Manual of cardiology. (Tilley, L.P., J.M. Owens, Eds). Churchill Livingstone, New York, 1986.

7. Lieb, A., J. Lang, C. W. Lombard. Case report: Eisenmenger syndrome in a 9-month-old Border collie puppy. Schweiz Arch Tierheilkd, 1998; 140, 164-167.

8. Nishida, K., Y. Wakao, T. Watanabe, M. Muto, T. Minami, Y. Ishikawa, T. Susuki, M. Takashi, M. Kawabata. A Case of Eisenmenger Syndrome of the Dog. J. of the Japan Vet. Med. Assoc., 1988; 41, 263-266.

9. Aiello, S.E., A. Mays, H. E. Amstutz, D. J. Anderson, S. J. Armour, L. B. Jeffcott, F. M. Loew, A. M. Wolf. The merck veterinary manual. Eighth edition. Merck\&Co Inc., Whitehouse station, USA, 1998.

10. Olivier, N.B. Congenital heart disease in the dog. In: Canine and feline cardiology. (Fox, P.R., Eds). Churchill Livingstone, New York, 1988; pp. 357-390.

11. Cebra, M., C. Cebra. Cardiovascular Diseases. In: Large Animal Internal Medicine. (Ogilvie, T.H., Eds), Williams and Wilkins Company, Baltimore, 1998; pp. 174-179.

12. Vodenicharov, A. Angiology. In: Anatomy of the domestic animals. Kovachev, G., G. Georgiev, A. Vodenicharov, Eds. 3d tome. Enyovche, Sofia, Bulgaria, 2004; pp. 26-28.

13. Fox, P.R. Congenital feline heart disease. In: Canine and feline cardiology. (Fox, P.R., Eds). Churchill Livingstone, New York, 1988; pp. 391-408.

14. Nakamura, K., M. Yamasaki, H. Ohta, N. Sasaki, M. Murikam, W.R. Bandula Kumara, M. Takiguchi. Effects of sildenafil citrate on five dogs with Eisenmenger's syndrome. J. Small Anim. Pract., 2011; 52, 595-598.

15. Chow, K.S., N. J. Bejerink, S. Ettinger, R. Flieger, J. A. Beatty, V. R. Barrs. Use of sildenafil citrate in a cat with Eisenmenger's syndrome and an atrial septal defect. Open Reports. J. of Feline Med. and Surg., 2015; 1-5.

\section{Corresponding author:}

Dr. Georgi I. Georgiev, DVM, PhD

Sofia, Bulgaria №1, Tsarigradsko shosse Blvd. 73 , tel: +359 898743055 email: dr_gigeorgiev@abv.bg 